TRANSACTIONS OF THE

AMERICAN MATHEMATICAL SOCIETY

Volume 361, Number 3, March 2009, Pages 1667-1681

S 0002-9947(08)04582-0

Article electronically published on October 23, 2008

\title{
MURRE'S CONJECTURES AND EXPLICIT CHOW-KÜNNETH PROJECTORS FOR VARIETIES WITH A NEF TANGENT BUNDLE
}

\author{
JAYA NN. IYER
}

\begin{abstract}
In this paper, we investigate Murre's conjectures on the structure of rational Chow groups and exhibit explicit Chow-Künneth projectors for some examples. More precisely, the examples we study are the varieties which have a nef tangent bundle. For surfaces and threefolds which have a nef tangent bundle, explicit Chow-Künneth projectors are obtained which satisfy Murre's conjectures, and the motivic Hard Lefschetz theorem is verified.
\end{abstract}

\section{INTRODUCTION}

Suppose $X$ is a nonsingular projective variety of dimension $n$ defined over the complex numbers. Let $C H^{i}(X) \otimes \mathbb{Q}$ be the Chow group of codimension $i$ algebraic cycles modulo rational equivalence. There has been a wide interest in understanding the structure of the Chow groups, and their study has led to many conjectural filtrations on the Chow groups. Jacob Murre [Mu2], Mu3] has made the following conjectures:

(A) The motive $h(X):=\left(X, \Delta_{X}\right)$ of $X$ has a Chow-Künneth decomposition:

$$
\Delta_{X}=\sum_{i=0}^{2 n} \pi_{i} \in C H^{n}(X \times X) \otimes \mathbb{Q}
$$

such that the $\pi_{i}$ are orthogonal projectors (see $\$ 2.2$ ). $\mathbb{Q}$.

(B) The correspondences $\pi_{0}, \pi_{1}, \ldots, \pi_{j-1}, \pi_{2 j+1}, \ldots, \pi_{2 n}$ act as zero on $C H^{j}(X) \otimes$

(C) Suppose

$$
F^{r} C H^{j}(X) \otimes \mathbb{Q}=\operatorname{Ker} \pi_{2 j} \cap \operatorname{Ker} \pi_{2 j-1} \cap \ldots \cap \operatorname{Ker} \pi_{2 j-r+1} .
$$

Then the filtration $F^{\bullet}$ of $C H^{j}(X) \otimes \mathbb{Q}$ is independent of the choice of the projectors $\pi_{i}$.

(D) Furthermore, $F^{1} C H^{i}(X) \otimes \mathbb{Q}=\left(C H^{i}(X) \otimes \mathbb{Q}\right)_{h o m}$, the cycles which are homologous to zero.

A related conjecture is that the motive of a smooth projective variety satisfies a motivic Hard Lefschetz theorem (see §2.5). A part of these conjectures has been verified for curves, surfaces, a product of a curve and surface Mu1, Mu3, abelian varieties and abelian schemes [Sh], De-Mu, uniruled threefolds [dA-Mü1], elliptic

Received by the editors November 6, 2006 and, in revised form, June 5, 2007.

2000 Mathematics Subject Classification. Primary 14C25, 14D05, 14D20, 14 D21.

Key words and phrases. Homogeneous spaces, Chow groups, projectors.

(C)2008 American Mathematical Society 
modular varieties [Go-Mu], GHM2]), universal families over Picard modular surfaces MWYK and finite group quotients (may be singular) of abelian varieties Ak-Jo. A criterion for the existence of a Chow-Künneth decomposition is also prescribed in $\mathrm{Sa}$.

On the other hand, S.I. Kimura Ki has introduced the notion of a finite dimensional motive. He showed the finite dimensionality of the motive of a curve and an abelian variety. He has conjectured that any nonsingular projective variety $X$ has a finite dimensional motive. Furthermore, if $X$ is known to have a Künneth decomposition, then finite dimensionality implies the existence of a Chow-Künneth decomposition for $X$ Gu-Pe, Corollary 9]. Hence Kimura's conjecture is related to Murre's conjectures.

Our aim here is to verify Murre's conjectures and exhibit explicit Chow-Künneth projectors, in the following situation. This also gives the existence of the conjectural filtration on the rational Chow groups for these varieties.

Theorem 1.1. Suppose $X$ is a nonsingular projective surface or a threefold which has a numerically effective tangent bundle. Then the motive of $X$ is finite dimensional and we have an explicit Chow-Künneth decomposition in terms of the projectors of its Albanese reduction. Moreover, Murre's conjectures are fulfilled by these projectors and the motivic Hard Lefschetz theorem holds.

See Corollary 3.14 and Proposition 3.16 in 3.4 for the explicit projectors.

Examples of varieties which have a nef tangent bundle include abelian varieties, hyperelliptic varieties and bundles of homogeneous varieties over an abelian variety.

A similar statement as in Theorem 1.1 holds for higher dimensional $(\geq 4)$ nonsingular varieties with a nef tangent bundle if we assume that homogeneous bundles $Z / S$ have a relative Chow-Künneth decomposition (in the sense of [De-Mu]) and any nonsingular Fano variety with a nef tangent bundle is a homogeneous variety; see 3.6

We note that our results imply that whenever a nonsingular projective variety $X$ admits a finite surjective cover $Z \longrightarrow X$ such that $Z \longrightarrow A$ is a relative cellular variety over an abelian variety $A$, then $X$ has an absolute Chow-Künneth decomposition and the consequences of Theorem 1.1 hold; see Corollary 3.7.

The proof of Theorem 1.1 involves the classification results ([Ca-Pe, [DPS]) and applying the criterion in GHM2 to obtain absolute Chow-Künneth projectors from relative Chow-Künneth projectors. We also write down the explicit ChowKünneth projectors for $X$ in terms of the projectors of its Albanese reduction (see Proposition 3.10). These projectors moreover satisfy Poincaré duality and part of Murre's conjectures (similar to the results on abelian varieties). Thus we apply the geometric methods and results on classification theory to answer some questions on algebraic cycles.

Here is an outline of the paper: we briefly review the preliminaries in $\S 2$. A study of the above conjectures for rational homogeneous bundles over varieties in some generality followed by a more detailed discussion for varieties with nef tangent bundle is carried out in $\S 3$.

\section{Preliminaries}

We work over the field of complex numbers in this paper and all the Chow groups are taken with $\mathbb{Q}$-coefficients. 
2.1. Category of motives. The category of nonsingular projective varieties over $\mathbb{C}$ will be denoted by $\mathcal{V}$. For an object $X$ of $\mathcal{V}$, let $C H^{i}(X)_{\mathbb{Q}}=C H^{i}(X) \otimes \mathbb{Q}$ denote the rational Chow group of codimension $i$ algebraic cycles modulo rational equivalence. We will use the standard framework of the category of Chow motives $\mathcal{M}_{\text {rat }}$ in this paper and refer to $\underline{\mathrm{Mu} 2}$ for details. We denote the category of motives $\mathcal{M}_{\sim}$, where $\sim$ is any equivalence; for instance $\sim$ is homological or numerical equivalence. When $S$ is a smooth variety, we also consider the category of relative Chow motives $C H \mathcal{M}(S)$ which is introduced in De-Mu and [GHM]. When $S=$ Spec $\mathbb{C}$, then the category $C H \mathcal{M}(S)=\mathcal{M}_{\text {rat }}$.

2.2. Chow-Künneth decomposition for a variety. Suppose $X$ is a nonsingular projective variety over $\mathbb{C}$ of dimension $n$. Let $\Delta_{X} \subset X \times X$ be the diagonal. Consider the Künneth decomposition of $\Delta$ in the Betti cohomology:

$$
\Delta_{X}=\bigoplus_{i=0}^{2 n} \pi_{i}^{h o m}
$$

where $\pi_{i}^{h o m} \in H^{2 n-i}(X) \otimes H^{i}(X)$.

Definition 2.1. The motive of $X$ is said to have Künneth decomposition if each of the classes $\pi_{i}^{h o m}$ is algebraic; i.e., $\pi_{i}^{h o m}$ is the image of an algebraic cycle $\pi_{i}$ under the cycle class map from the rational Chow groups to the Betti cohomology.

Definition 2.2. The motive of $X$ is said to have a Chow-Künneth decomposition if each of the classes $\pi_{i}^{h o m}$ is algebraic and the $\pi_{i}$ are orthogonal projectors, i.e., $\pi_{i} \circ \pi_{j}=\delta_{i, j} \pi_{i}$.

We recall the following well-known statement which we will need in Proposition 3.10 .

Lemma 2.3. Suppose $f: X \longrightarrow Y$ is a finite surjective morphism. If $X$ has a Künneth decomposition, then $Y$ also has a Künneth decomposition.

Proof. Suppose $\pi_{i}^{X}$ are the Künneth components for $X$. Since $f_{*} \Delta_{X}=m \cdot \Delta_{Y}$, where $m=\operatorname{degree}(f)$, it follows that $(1 / m) \cdot f_{*}\left(\pi_{i}^{X}\right)$ are the Künneth components for $Y$.

2.3. Relative Chow-Künneth decomposition. Suppose $f: X \longrightarrow S$ is a smooth projective morphism of relative dimension $d$. A relative Chow-Künneth decomposition of $X \longrightarrow S$ is a decomposition of the relative diagonal cycle

$$
\Delta_{S}=\sum_{i=0}^{2 g} \Pi_{i} \in C H^{d}\left(X \times_{S} X\right) \otimes \mathbb{Q}
$$

such that $\Pi_{i} \circ \Pi_{j}=\delta_{i, j} \Pi_{i}$; i.e., they are orthogonal projectors.

It is conjectured that any smooth projective morphism as above admits a relative Chow-Künneth decomposition and instead of the cohomology, the projectors act on $\mathbf{R} f_{*} \mathbb{Q}$ in the derived category of bounded complexes of cohomologically constructible $\mathbb{Q}$-sheaves on $S$ (see $[\mathrm{De}-\mathrm{Mu}$ ). More generally, it is conjectured by Corti and Hanamura $\mathrm{Co}-\mathrm{Ha}$, that a motivic decomposition theorem is true for a stratified projective morphism.

We recall the criterion in GHM2 to obtain absolute Chow-Künneth projectors from the relative Chow-Künneth projectors. We will need this in $₫ 3.2$. Since we will 
be dealing with a smooth projective morphism $\pi: X \rightarrow S$, we state the criterion in GHM2 in this case.

We first state the condition to have an explicit decomposition.

Lemma 2.4. Let $X$ and $S$ be smooth quasi-projective varieties over $\mathbb{C}$ and $\pi: X \rightarrow$ $S$ be a projective morphism. Then

a) giving a projector $\Pi \in C H_{\operatorname{dim}{ }_{X}}\left(X \times_{S} X\right)$ and an isomorphism in $C H \mathcal{M}(S)$

$$
f:(X, \Pi, 0) \longrightarrow \oplus^{m}(S, \Delta(S),-q)
$$

is equivalent to giving elements $f_{1}, \ldots, f_{m} \in C H_{\operatorname{dim} X+q}(X)$ and elements $g_{1}, \ldots, g_{m} \in$ $\mathrm{CH}_{\text {dim } \mathrm{X}_{-q}}(\mathrm{X})$ subject to the condition $\pi_{*}\left(f_{i} . g_{j}\right)=\delta_{i j}[S]$. Here $f_{i} . g_{j}$ is the intersection product in $X$ and $[S] \in \mathrm{CH}_{\operatorname{dim}}{ }_{S}(S)$ is the fundamental class.

b) If $(X, P, 0) \in C H M(S)$ and $\Pi$ is a constituent of $P$ (i.e., $P \circ \Pi=\Pi \circ P=\Pi)$, then we can take the above $f_{i}$ and $g_{j}$ such that moreover $f_{i} \circ P=f_{i}$ and $g_{j}=P \circ g_{j}$. Conversely if we have such $f_{i}$ and $g_{j}$, then the corresponding $\Pi$ is a constituent of $P$.

Proof. This is [GHM2, Lemma 4].

Theorem 2.5. Suppose the variety $\pi: X \rightarrow S$ has a relative Chow-Künneth decomposition. This means that there is an orthogonal set of projectors $P^{i} \in$ $C H_{\operatorname{dimX}}\left(X \times_{S} X\right)$ with $\sum_{i} P^{i}=1$ such that $P^{i}$ acts on $R^{j} \pi_{*} \mathbb{Q}_{X}$ as identity if $j=i$ and as zero if $j \neq i$. Assume the following conditions:

1. S has a Chow-Künneth decomposition over $\mathbb{C}$.

2. If $t$ is a point of $S$, the natural map

$$
C H^{r}(X) \longrightarrow H_{B}^{2 r}\left(X_{t}(\mathbb{C}), \mathbb{Q}\right)^{\pi_{1}^{t o p}(S, t)}
$$

is surjective for $0 \leq r \leq d:=\operatorname{dimX} / S$. The target is the invariant part under the action of the topological fundamental group of $S$.

3. For $i$ odd, $H_{B}^{i}\left(X_{t}, \mathbb{Q}\right)^{\pi_{1}^{t o p}(S, t)}=0$.

4. If $\mathcal{V}$ is the local system $R^{i} \pi_{*} \mathbb{Q}_{X}$, for any $i=2 r-1,0 \leq r \leq d$, or the local system $R^{2 r} \pi_{*} \mathbb{Q}_{X} /\left(R^{2 r} \pi_{*} \mathbb{Q}_{X}\right)^{\pi_{1}}$, if $0 \leq r \leq d$, then $H^{q}(S, \mathcal{V})=0$ if $q \neq \operatorname{dim} S$. $\mathbb{C}$.

Under these assumptions $X$ has an absolute Chow-Künneth decomposition over

Proof. This is GHM2, Main Theorem 1.3]. In loc. cit the local system in assumption 4 is arbitrary but we notice that in the proof only those local systems as stated above are required to satisfy assumption 4 .

Lemma 2.6. Construction of a projector $\left(P^{2 r} / S\right)_{\text {alg }}$ (the subscript "alg" indicates it splits the algebraic cohomology in the fiber $X_{t}$ ) which is a constituent of a projector $P^{2 r} / S$, together with an isomorphism

$$
\left(X / S,\left(P^{2 r} / S\right)_{a l g}, 0\right) \simeq \oplus^{m}(S, \Delta(S),-r)
$$

follows from assumption 2 of Theorem 2.5 .

Proof. This is proved in Step II in the proof of [GHM2, Main Theorem].

We will apply these statements when we look at rational homogeneous bundles over abelian varieties in $\$ 3.2$. 
2.4. Finite dimensional motives. The notion of a finite dimensional motive was introduced by S.-I. Kimura. We refer to his paper [Ki] for the definition and properties. Examples of finite dimensional motives include the case of curves and abelian varieties. We recall the following results from [Ki] which we will use in this paper. These provide further examples of finite dimensional motives.

Lemma 2.7 ([Ki, Corollary 5.11]). If the motives of $X$ and $Y$ are finite dimensional, then the motive of the product $X \times Y$, the sum $X \oplus Y$ and a direct summand of a finite dimensional motive are also finite dimensional.

Lemma 2.8 ([Ki, Proposition 6.9]). Suppose $f: X \longrightarrow Y$ is a surjective morphism. If the motive of $X$ is finite dimensional, then the motive of $Y$ is also finite dimensional.

Kimura ([Ki, Proposition 7.5]) observed that the finite dimensionality of a motive $M$ implies the nilpotency of the ideal $I$ defined by the exact sequence

$$
0 \longrightarrow I \longrightarrow \operatorname{End}_{\mathcal{M}_{\text {rat }}}(M) \longrightarrow \operatorname{End}_{\mathcal{M}_{\text {hom }}}(M) \longrightarrow 0 \text {. }
$$

A result due to U. Jannsen ( $[\mathrm{Ja}, 5.3]$ ) is used to deduce the following:

Proposition 2.9 ( $[\mathrm{Gu}-\mathrm{Pe}$, Corollary 9]). Suppose $h(X)$ is finite dimensional. Assume that the Künneth components of $X$ are algebraic. Then $X$ has a ChowKünneth decomposition.

2.5. Lefschetz decomposition of a motive. We recall the definitions of Lefschetz operator and Lefschetz decomposition of motives. We refer to $[\mathrm{Kl}, \mathrm{Ku}$, for the details.

Suppose we are given a smooth projective variety $X$ of dimension $n$ and an ample divisor $\alpha \in C H^{1}(X)_{Q}$. We can define the Lefschetz operator $L_{\alpha}$. Consider the closed embedding $i: \alpha \hookrightarrow X$. Define

$$
L_{\alpha}:=i_{*} i^{*}: h(X) \longrightarrow h(X)(1) .
$$

Then the $(n-1)$-fold composition with itself is denoted by

$$
L_{\alpha}^{n-i}: h(X) \longrightarrow h(X)(n-i) .
$$

In the realizations $L_{\alpha}$ induces the multiplication with the class of $\alpha$. Then the Hard Lefschetz theorem gives an isomorphism between the cohomology groups defined by the Lefschetz operator:

$$
L_{\alpha}^{n-i}: H^{i}(X, \mathbb{Q}) \stackrel{\simeq}{\longrightarrow} H^{2 n-i}(X, \mathbb{Q}) .
$$

Furthermore, there is a decomposition of the cohomology into primitive cohomologies:

$$
H^{i}(X, \mathbb{Q})=\bigoplus_{\max \{0, i-n\} \leq k \leq[i / 2]} L^{k} P^{i-2 k}(X, \mathbb{Q}) .
$$

The standard conjectures of Lefschetz type predict a motivic statement of the above statements which says that there is an isomorphism of motives

$$
L_{\alpha}^{n-i}: h^{i}(X) \stackrel{\simeq}{\longrightarrow} h^{2 n-i}(X)(n-i)
$$

and a decomposition of motives

$$
h^{i}(X)=\bigoplus_{\max \{0, i-n\} \leq k \leq[i / 2]} L_{\alpha}^{k} P^{i-2 k}(X) .
$$

Here $P^{i}(X)$ is the motivic analogue of the primitive cohomology. 
Theorem 2.10. Suppose $X$ is an abelian variety. Then the motivic Hard Lefschetz theorem and the Lefschetz decomposition hold for $X$.

Proof. This was proved by Künnemann and Scholl; see $[\mathrm{Ku}$, $[\mathrm{Sc}$.

\section{RATIONAL HOMOGENEOUS BUNDLES OVER A VARIETY AND VARIETIES WITH A NEF TANGENT BUNDLE}

In this section we begin by recalling the motive of a homogeneous space and of a relative cellular variety. These results will be used to study the motive of varieties with a numerically effective tangent bundle.

3.1. The motive of a rational homogeneous space. Let $G$ be a reductive linear algebraic group and $P$ be a parabolic subgroup of $G$. Then $F:=G / P$ is a complete variety. Notice that $F$ is a cellular variety; i.e., it has a cellular decomposition

$$
\emptyset=F_{-1} \subset F_{0} \subset \ldots \subset F_{n}=F
$$

such that each $F_{i} \subset F$ is a closed subvariety and $F_{i}-F_{i-1}$ is an affine space.

Then we have

Lemma 3.1 ([Ko, Theorem, p. 363]). The Chow motive $h(F)=\left(F, \Delta_{F}\right)$ of $F$ decomposes as a direct sum of twisted Tate motives

$$
h(F)=\bigoplus_{\omega} \mathbb{L}^{\otimes \operatorname{dim} \omega} .
$$

Here $\omega$ runs over the set of cells of $F$.

3.2. The motive of a rational homogeneous bundle. Consider a rational homogeneous bundle

$$
f: Z \longrightarrow Y \text {. }
$$

In other words, $f$ is a smooth projective morphism and any fiber $f^{-1} y$ is a rational homogeneous variety $G / P$, for some reductive linear algebraic group $G$ and a parabolic subgroup $P$ of $G$.

If $Z \longrightarrow Y$ is a relative cellular variety, then it might be possible to prove the following lemma directly, as mentioned to us by R. Joshua. We consider a more general situation when we have a homogeneous bundle and, under the assumption of existence, of a relative Chow-Künneth decomposition. We apply the criterion in GHM2 to deduce an absolute Chow-Künneth decomposition.

Lemma 3.2. Let $Y$ be a smooth variety which has a Chow-Künneth decomposition. Suppose $Z \longrightarrow Y$ is a rational homogeneous bundle and $d:=\operatorname{dim} Z-\operatorname{dim} Y$. Assume that the bundle $Z \longrightarrow Y$ has a relative Chow-Künneth decomposition in the category of relative Chow motives $C H \mathcal{M}(Y)$ over $Y$. Then the absolute motive of $Z$ also admits a Chow-Künneth decomposition.

Proof. By assumption, we have relative Chow-Künneth projectors for the variety $Z \longrightarrow Y$. Write a relative Chow-Künneth decomposition of the relative Chow motive of $Z / Y$ as

$$
(Z / Y, 1,0)=\bigoplus_{r=0}^{2 d}\left(Z / Y, \Pi_{r}, 0\right)
$$

in $C H \mathcal{M}(Y)$. We want to apply Theorem 2.5 to deduce the absolute ChowKünneth decomposition from the relative Chow-Künneth decomposition. For this 
purpose we consider the ordinary rational cohomology of the fibres of $f$ and show that the cohomology classes of the fibres are invariant under the topological fundamental group of the base $Y$ under the natural monodromy action. More precisely, the fibres of $f$ are smooth homogeneous varieties and have only algebraic cohomology. Hence for any $t \in Y$, it follows that the map

$$
C H^{r}(Z) \longrightarrow H_{B}^{2 r}\left(Z_{t}, \mathbb{Q}\right)^{\pi_{1}^{t o p}(Y, t)}
$$

is surjective for $0 \leq r \leq d$, and for $i$ odd,

$$
H_{B}^{i}\left(Z_{t}, \mathbb{Q}\right)^{\pi_{1}^{t o p}(Y, t)}=0 .
$$

Hence the assumptions 1, 2, and 3 in Theorem 2.5 are fulfilled. Moreover the local systems $R^{i} \pi_{*} \mathbb{Q}_{Z}$, for any $i=2 r-1,0 \leq r \leq d$, or $R^{2 r} \pi_{*} \mathbb{Q}_{Z} /\left(R^{2 r} \pi_{*} \mathbb{Q}_{Z}\right)^{\pi_{1}}$, if $0 \leq r \leq d$, are vanishing. The assumption 4 in Theorem 2.5 is now also satisfied. Applying Theorem 2.5. we obtain an absolute Chow-Künneth decomposition for $Z$.

Corollary 3.3. With notation and assumptions as in Lemma 3.2, the motive of $Z$ is

$$
\left(Z, \Delta_{Z}\right)=\bigoplus_{i}\left(Z, \pi_{i}^{Z}\right)
$$

where $\left(Z, \pi_{i}^{Z}\right)=\bigoplus_{j+k=i} m_{j} \cdot \mathbb{L}^{j} \otimes\left(Y, \pi_{k}^{Y}\right)$. Here $m_{j}$ is the number of $j$-codimensional cells on a fiber $\mathbb{F}$ and $\left(Y, \Delta_{Y}\right)=\bigoplus_{k}\left(Y, \pi_{k}^{Y}\right)$ is a Chow-Künneth decomposition for $Y$.

Proof. Now applying Lemma 2.6 we get an isomorphism of the relative Chow motives

$$
\left(Z / Y, \Pi_{2 r}, 0\right) \simeq(Y, 1,-r)^{\oplus m_{r}}
$$

in $C H \mathcal{M}(Y)$, where $m_{r}=\operatorname{dim} H_{B}^{2 r}\left(Z_{t}, \mathbb{Q}\right)$. Using [GHM2, Lemma 1] the natural map

$$
\phi: C H_{\operatorname{dim} Z}\left(Z \times_{Y} Z\right) \longrightarrow C H_{\operatorname{dim} Z}\left(Z \times_{\mathbb{C}} Z\right)
$$

is a ring homomorphism and hence transforms orthogonal projectors over $Y$ to orthogonal projectors over $\mathbb{C}$. Hence we have a functor

$$
\begin{aligned}
\Phi: C H \mathcal{M}(Y) & \longrightarrow \mathcal{M}_{\text {rat }}(\mathbb{C}) \\
\left(Z / Y, \Pi_{2 r}, i\right) & \mapsto \quad\left(Z, P_{2 r}, i\right) .
\end{aligned}
$$

Since the cohomology of the fibres $Z_{t}$ is algebraic, by GHM2, $\S 1.7$, Step V], there is an isomorphism

$$
\left(Z, P_{2 r}, 0\right) \simeq(Y, 1,-r)^{\oplus m_{r}}
$$

Since $Y$ has a Chow-Künneth decomposition we can write this decomposition as

$$
\left(Y, \Delta_{Y}\right)=\bigoplus_{l}\left(Y, \pi_{l}^{Y}\right)
$$

This gives

$$
\begin{aligned}
\left(Z, \Delta_{Z}\right) & =\bigoplus_{r=0}^{d}\left(Z, P_{2 r}\right) \\
& =\bigoplus_{r=0}^{d} \oplus_{k} m_{r} \cdot \mathbb{L}^{r} \otimes\left(Y, \pi_{k}^{Y}\right) .
\end{aligned}
$$


Write

$$
\left(Z, \pi_{i}^{Z}\right):=\bigoplus_{j+k=i} m_{j} \cdot \mathbb{L}^{j} \otimes\left(Y, \pi_{k}^{Y}\right)
$$

to give a Chow-Künneth decomposition of $\left(Z, \Delta_{Z}\right)=\bigoplus_{i}\left(Z, \pi_{i}^{Z}\right)$.

Corollary 3.4. With notation and assumptions as in Lemma 3.2, the motive of $Z$ is finite dimensional if the motive of $Y$ is finite dimensional.

Proof. This follows by Lemma 2.7 and Corollary 3.3

Corollary 3.5. Suppose $Z \longrightarrow Y$ is a homogeneous bundle which has a relative cellular decomposition. If the motive of $Y$ is finite dimensional, then the motive of $Z$ is also finite dimensional.

Proof. By [Ko and $\mathrm{Ne}-\mathrm{Za}$, we know that the motive of $Y$ has a relative ChowKünneth decomposition over $Z$. So we can apply Corollary 3.3 and Corollary 3.4 .

Fix an ample divisor $\alpha \in C H^{1}(Z)_{\mathbb{Q}}$ which is the pullback of an ample divisor $\beta \in C H^{1}(Y)_{\mathbb{Q}}$ twisted by a relatively ample divisor $\gamma \in C H^{1}(Z)$. Consider the Lefschetz operator $L_{\alpha} \in C H^{*}(Z \times Z)_{\mathbb{Q}}$, as defined in $\$ 2.5$, Let $m=\operatorname{dim} Y$, $f=\operatorname{dim} \mathbb{F}$ and $n=m+f$. Here $\mathbb{F}$ is a fiber of $Z \rightarrow Y$.

Corollary 3.6. Suppose that the motivic Hard Lefschetz theorem and the Lefschetz decomposition hold for $Y$. Then they also holds for the variety $Z$.

Proof. By Corollary 3.3, we can express the motive $h^{i}(Z)$ as $\bigoplus_{j+k=i} m_{j} . \mathbb{L}^{j} \otimes h^{k}(Y)$. Here $m_{j}$ is the number of $j$-codimensional cells on $\mathbb{F}$. Hence

$$
L_{\alpha}^{n-i}=\bigoplus_{j+k=i} m_{j} . L_{\gamma}^{f-j} \otimes L_{\beta}^{m-k}
$$

and

$$
L_{\alpha}^{n-i}: h^{i}(Z) \longrightarrow h^{2 n-i}(Z)
$$

gives an isomorphism. A similar argument holds for the Lefschetz decomposition.

3.3. Varieties with a nef tangent bundle. In this subsection, we want to deduce the properties discussed in $\S 3.2$ for varieties with a nef tangent bundle. We want to use the classification results by Campana and Peternell to describe the motive of these varieties.

We first prove the following statement, which holds for some wider class of varieties, and later deduce some consequences for varieties with a nef tangent bundle.

Lemma 3.7. Suppose $X$ is a nonsingular projective variety such that there is a finite surjective cover $Z \longrightarrow X$ and $Z$ admits a fibration

$$
\pi: Z \longrightarrow A
$$

which is a relative cellular variety over an abelian variety $A$. Then $X$ has a ChowKünneth decomposition and is finite dimensional. Furthermore, the motivic Hard Lefschetz theorem and the Lefschetz decomposition hold for X. 
Proof. Using Lemma 3.2 and Corollary 3.4 we conclude that $Z$ has a finite dimensional motive and a Chow-Künneth decomposition. Now by Lemma 2.3 and Lemma 2.7, we deduce that the variety $X$ has a finite dimensional motive and a Künneth decomposition. Again applying Proposition 2.9, we conclude that $X$ has a Chow-Künneth decomposition.

Since $g$ is a finite morphism, we consider an ample divisor $\alpha$ on $X^{\prime}$ which is the pullback of an ample divisor $\eta$ on $X$. By [Ki, Remark 10.6], the motive of $A$ is finite dimensional and $A$ has a Chow-Künneth decomposition [Sh]. Also the motivic Hard Lefschetz theorem and the Lefschetz decomposition hold for the abelian variety $A$, by Theorem 2.10,

Hence, applying Corollary [3.6. the Lefschetz operator $L_{\alpha}$ induces isomorphisms

$$
L_{\alpha}^{n-i}: h^{i}\left(X^{\prime}\right) \stackrel{\simeq}{\longrightarrow} h^{2 n-i}\left(X^{\prime}\right) \text {. }
$$

Since the motive $h^{i}(X)$ is a direct summand of the motive $h^{i}\left(X^{\prime}\right)$, the operator $L_{\alpha}^{n-i}$ restricts on $h^{i}(X)$ to the operator $L_{\eta}^{n-i}$. This gives a motivic Hard Lefschetz theorem for $X$. A similar argument also holds for the Lefschetz decomposition.

Corollary 3.8. Suppose $X$ is a nonsingular projective variety with numerically effective tangent bundle such that there is an étale cover $X^{\prime}$ which is a relative cellular variety over an abelian variety. Then the motive of $X$ is finite dimensional and has a Chow-Künneth decomposition. Furthermore, the motivic Hard Lefschetz theorem and Lefschetz decomposition hold for $X$.

We will see in $\$ 3.5$, that the assumptions made in the statements of Corollary 3.8 are fulfilled by surfaces and threefolds with a nef tangent bundle and hence the above consequences hold for such varieties.

3.4. Chow-Künneth projectors. Suppose $A$ is an abelian variety with a finite group $G$ acting on $A$. Then we have

Theorem 3.9. Let $A$ be an abelian variety of dimension $d$ and $G$ be a finite group acting on $A$. Let $h: A \longrightarrow A / G$ be the quotient morphism. Suppose $\Delta_{A}=\sum_{i=0}^{2 d} \pi_{i}$ is the Deninger-Murre Chow-Künneth decomposition for $A$ and let

$$
\eta_{i}=\frac{1}{|G|}(h \times h)_{*} \pi_{i} .
$$

Then the Chow-Künneth decomposition for $A / G$ is

$$
\Delta_{\frac{A}{G}}=\sum_{i=0}^{2 d} \eta_{i}
$$

This Chow-Künneth decomposition satisfies Poincaré duality, i.e., $\eta_{2 d-i}={ }^{t} \eta_{i}$, for any $i$.

In addition, $\eta_{i}$ acts as zero on $C H^{j}(A / G)_{\mathbb{Q}}$ for $i<j$ and also for $i>j+d$ in general. In the case $d \leq 4$, we conclude that $\eta_{i}$ acts trivially on $C H^{j}(A / G)_{Q}$ for $i<j$ and for $i>2 j$.

Proof. This is [De-Mu, Theorem 3.1] when $G$ is trivial, and when $G$ is nontrivial this is Ak-Jo, Theorem 1.1].

Suppose $X$ is a nonsingular projective variety with numerically effective tangent bundle. By [DPS], there is a finite étale cover

$$
q: X^{\prime} \longrightarrow X
$$


such that $X^{\prime}$ admits a fibration

$$
f^{\prime}: X^{\prime} \longrightarrow A^{\prime}
$$

where $A^{\prime}$ is an abelian variety and the fibres of $f^{\prime}$ are Fano varieties with a nef tangent bundle. We will assume that such Fano varieties are rational homogeneous spaces (see 33.6 ).

Since the fibers $F$ of $f^{\prime}$ are simply connected and are rationally connected varieties [Kol], KMM], the homogeneous space $F$ does not admit any nonconstant morphisms to an abelian variety (as there are no nonconstant morphisms from $\mathbb{P}^{1}$ to an abelian variety). Hence, there is a covering map $q^{\prime}: A^{\prime} \rightarrow A$ and a surjective morphism $f: X \rightarrow A$, such that $q$ is the pullback of $q^{\prime}$ by $f$. Now, any morphism between abelian varieties is a composition of a group homomorphism and a translation by an element [La-Bi, Proposition 2.1 a)]. Since a translation map is an isomorphism and induces isomorphisms on the Chow groups, without any confusion, we assume that $q^{\prime}$ is a surjective group homomorphism. Let $G$ be the kernel of $q^{\prime}$. Then $A=A^{\prime} / G$ and $q^{\prime}: A^{\prime} \longrightarrow A=A^{\prime} / G$.

Moreover, we have a commutative diagram

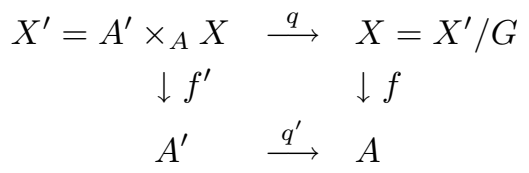

such that $G$ acts on the first factor of $X^{\prime}$ and has a trivial action on the second factor.

Next, we want to obtain explicit Chow-Künneth projectors for the variety $X$ in terms of the projectors of its Albanese reduction.

Proposition 3.10. Let $\Delta_{A}=\sum_{i} \pi_{i}^{A}$ be the Deninger-Murre Chow-Künneth decomposition for $A$. Then $\Delta_{X}=\sum_{i} \pi_{i}^{X}$ where

$$
\pi_{i}^{X}=\sum_{j+k=i} \tilde{\pi}_{k} \cdot(f \times f)^{*} \pi_{j}^{A}
$$

is a Chow-Künneth decomposition for $X$. Here $\left\{\tilde{\pi}_{k}\right\}$ are the images of the projectors defined on the cover $X^{\prime}$ as in Lemma 3.3. Here $d=\operatorname{dim} A$ and $n=\operatorname{dim} F$.

Proof. Firstly, we claim that the (rational) pushforward of the Deninger-Murre Chow-Künneth decomposition $\Delta_{A^{\prime}}$ is the Deninger-Murre Chow-Künneth decomposition on $A$; by Lemma 2.3, the (rational) pushforward of a Chow-Künneth decomposition of $\Delta_{A^{\prime}}$ is a Künneth decomposition. Consider the multiplication map, for any $n \in \mathbb{Z}$,

$$
i d \times n: A^{\prime} \times A^{\prime} \longrightarrow A^{\prime} \times A^{\prime} .
$$

There is an eigenspace decomposition of the rational Chow groups of $A^{\prime} \times A^{\prime}$. These eigenspaces are defined in De-Mu, Corollary 2.21]. We further apply Be, Proposition $2 \mathrm{c}$ )] to obtain that the pushforward map $\left(q^{\prime} \times q^{\prime}\right)_{*}$ respects the eigenspace decomposition of the Chow groups of $A^{\prime} \times A^{\prime}$ and $A \times A$. In particular, consider the Deninger-Murre Chow-Künneth decomposition

$$
\Delta_{A^{\prime}}=\sum_{i=0}^{2 d} \pi_{i}
$$


such that $(i d \times n)^{*} \pi_{i}=n^{i} \pi_{i}$, for all $n \in \mathbb{Z}$. Furthermore, this is a unique decomposition with these properties [De-Mu, Theorem 3.1].

Then the (rational) pushforward to $A \times A$ of the above decomposition of $\Delta_{A^{\prime}}$ also satisfies the same properties and, by the uniqueness of such a decomposition, gives the Deninger-Murre Chow-Künneth decomposition of $A$.

Consider the Chow-Künneth decomposition of $X^{\prime}$, using Corollary 3.3 ,

$$
\left(X^{\prime}, \Delta_{X^{\prime}}\right)=\bigoplus_{i}\left(X^{\prime}, \pi_{i}^{X^{\prime}}\right),
$$

where $\left(X^{\prime}, \pi_{i}^{X^{\prime}}\right)=\bigoplus_{j+k} m_{j} \cdot \mathbb{L}^{j} \otimes\left(A^{\prime}, \pi_{k}^{A^{\prime}}\right)$. Here $m_{j}$ is the number of $j$-codimensional cells on a fiber $\mathbb{F}$ and $\pi_{k}^{A^{\prime}}$ are the Deninger-Murre Chow-Künneth projectors of $A^{\prime}$.

Now consider the (rational) pushforward $(q \times q)_{*}$ of this Chow-Künneth decomposition of $X^{\prime}$, which is now a Künneth decomposition of $X$, by Lemma 2.3 . Since the pushforwards of the projectors $\pi_{k}^{A^{\prime}}$ are precisely the Deninger-Murre Chow-Künneth projectors of $A$ (from the above discussion), we deduce that the pushforwards of the projectors $\pi_{i}^{X^{\prime}}$ satisfy the orthogonality properties.

Suppose $X^{\prime} \longrightarrow A^{\prime}$ is a relative cellular space.

Then there is a sequence of closed embeddings

$$
\emptyset=Z_{-1} \subset Z_{0} \subset \ldots \subset Z_{n}=X^{\prime}
$$

such that $\pi_{k}: Z_{k} \longrightarrow Y$ is a flat projective $A^{\prime}$-scheme. Further, for any $k=$ $0,1, \ldots, n$, the open complement $Z_{k}-Z_{k-1}$ is $A^{\prime}$-isomorphic to an affine space $\mathbb{A}_{A^{\prime}}^{m_{k}}$ of relative dimension $m_{k}$. Denote $i_{k}: Z_{k} \hookrightarrow Z$.

Then we have

Lemma 3.11. For any $a, b \in \mathbb{Z}$, the map

$$
\begin{gathered}
\bigoplus_{k=0}^{n} H_{a-2 m_{k}}\left(A^{\prime}, b-m_{k}\right) \longrightarrow H_{a}\left(X^{\prime}, b\right) \\
\left(\alpha_{0}, \ldots, \alpha_{n}\right) \mapsto \sum_{k=0}^{n}\left(i_{k}\right)_{*} \pi_{k}^{*} \alpha_{k}
\end{gathered}
$$

is an isomorphism. Here $H_{a}\left(A^{\prime}, b\right)=C H_{b}\left(A^{\prime}, a-2 b\right)$, the higher Chow groups of $A^{\prime}$.

Proof. See [Ko, Theorem, p. 371].

Remark 3.12. The above Lemma 3.11 can equivalently be restated to express the Chow groups of $X^{\prime}$ as

$$
C H^{r}\left(X^{\prime}\right)_{\mathbb{Q}}=\bigoplus_{k=0}^{r}\left(\sum_{\alpha} \mathbb{Q}\left[\omega_{k}^{\alpha}\right]\right) \cdot f^{*} C H^{k}\left(A^{\prime}\right)_{\mathbb{Q}} .
$$

Here $\omega_{k}^{\alpha}$ are the $(r-k)$-codimensional relative cells and $\alpha$ runs over the indexing set of $(r-k)$-codimensional relative cells in the $A^{\prime}$-scheme $X^{\prime}$.

Lemma 3.13. Suppose $X$ is a nonsingular projective variety and has a finite étale cover $X^{\prime} \longrightarrow A$ which is a relative cellular variety over an abelian variety $A$. Then 
the Chow groups of $X$ can be written as

$$
C H^{r}(X)_{\mathbb{Q}}=\bigoplus_{k=0}^{r}\left(\sum_{\alpha} \mathbb{Q} \cdot\left[\bar{\omega}_{k}^{\alpha}\right]\right) \cdot f^{*} C H^{k}(A)_{\mathbb{Q}} .
$$

Here $\bar{\omega}_{k}^{\alpha}$ are the images of the $(r-k)$-codimensional relative cells $\omega_{k}^{\alpha}$ and $\alpha$ runs over the indexing set of $(r-k)$-codimensional relative cells in the $A^{\prime}$-scheme $X^{\prime}$ (see Remark 3.12).

Proof. Using [Fu, Example 1.7.6, p. 20], the Chow groups of $X$ are written as

$$
\begin{aligned}
C H^{r}(X)_{\mathbb{Q}} & =\left(C H^{r}\left(X^{\prime}\right)_{\mathbb{Q}}\right)^{G}, \text { the group of } G \text {-invariants in } C H^{r}\left(X^{\prime}\right)_{\mathbb{Q}} \\
& =\bigoplus_{k=0}^{r}\left(\sum_{\alpha} \mathbb{Q}\left[\omega_{k}^{\alpha}\right]\right) \cdot\left(f^{\prime *} C H^{k}\left(A^{\prime}\right)_{\mathbb{Q}}\right)^{G}, \text { by Remark } 3.12 \\
& =\bigoplus_{k=0}^{r}\left(\sum_{\alpha} \mathbb{Q}\left[\omega_{k}^{\alpha}\right]\right) \cdot f^{\prime *}\left(C H^{k}\left(A^{\prime}\right)_{\mathbb{Q}}^{G}\right) \\
& =\bigoplus_{k=0}^{r}\left(\sum_{\alpha} \mathbb{Q}\left[\bar{\omega}_{k}^{\alpha}\right]\right) \cdot f^{*} C H^{k}(A)_{\mathbb{Q}}, \text { again by loc. cit. }
\end{aligned}
$$

Corollary 3.14. The Chow-Künneth decomposition obtained in Proposition 3.10 satisfies Poincaré duality, i.e., $\pi_{2 d-i}^{X}={ }^{t} \pi_{i}^{X}$, for any $i$. In addition, $\pi_{i}^{X}$ acts as zero on $C H^{j}(X)_{\mathbb{Q}}$ for $i<j$ and also for $i>j+d+n$. In the case $d \leq 4$, we conclude that $\pi_{i}^{X}$ acts trivially on $C H^{j}(X)_{Q}$ for $i<j$ and for $i>2 j$.

Proof. To prove the assertion, notice that the action of the projectors $\pi_{i}^{X}$ is given by the projectors $\tilde{\pi_{j}}$ (respectively $\left.\pi_{k}^{A}\right)$ on $\left(\sum_{\alpha} \mathbb{Q}\left[\omega_{k}^{\alpha}\right]\right.$ ) (respectively on $C H^{k}(A)_{\mathbb{Q}}$; for instance, see Theorem (3.9). Since the assertion is true for the projectors $\tilde{\pi}_{j}$ and $\pi_{k}^{A}$ in the desired range, the assertion is true for the projectors $\pi_{i}^{X}$.

3.5. Surfaces and threefolds with a nef tangent bundle. In this subsection we want to show that surfaces and threefolds with a nef tangent bundle satisfy Murre's conjectures.

For this purpose, we recall the classification theorem of such varieties proved by Campana and Peternell.

Theorem 3.15 (Ca-Pe, Theorem 10.1]). Suppose $X$ is a nonsingular projective threefold. Then the following are equivalent:

1. The tangent bundle $T_{X}$ is nef.

2. Some étale covering $X^{\prime}$ of $X$ belongs to the following list:

a) $X^{\prime}=X$ and is a nonsingular quadric or is the projective space (i.e., $X$ is rational homogeneous and $\left.b_{2}(X)=1\right)$.

b) $X^{\prime}=X=\mathbb{P}\left(T_{X}\right)$ (i.e., $X$ is rational homogeneous and $b_{2}(X)>1$ ).

c) $X^{\prime}=\mathbb{P}(E)$ for a flat rank 3 vector bundle $E$ on an elliptic curve.

d) $X^{\prime}=\mathbb{P}(F) \times_{C} \mathbb{P}\left(F^{\prime}\right)$ for flat rank 2 vector bundles $F$ and $F^{\prime}$ over an elliptic curve $C$.

e) $X^{\prime}=\mathbb{P}(E)$ for a flat rank 2 vector bundle $E$ on an abelian surface.

f) $X^{\prime}$ is an abelian threefold. 
Proposition 3.16. Suppose $X$ is a nonsingular projective surface or a threefold which has a nef tangent bundle. Then the motive of $X$ is finite dimensional and we have an explicit Chow-Künneth decomposition in terms of the projectors of its Albanese reduction. Moreover, Murre's conjectures are fulfilled by these projectors as in Corollary 3.14 and the motivic Hard Lefschetz theorem holds.

Proof. We only need to show that there is an étale cover $X^{\prime}$ of $X$ which is a relative cellular variety over an abelian variety. We now apply Theorem 3.15 to deduce that there is an étale cover $X^{\prime}$ of $X$ which is either a projectivization $\mathbb{P}(E)$ of a vector bundle $E$ over an abelian variety $A$ or a product of such varieties over an abelian variety. Since the projectivization $\mathbb{P}(E)$ is clearly a relative cellular variety over $A$, the same is true of a product $\mathbb{P}(E) \times_{C} \mathbb{P}\left(E^{\prime}\right)$ over an elliptic curve. A similar classification result holds for surfaces; see Ca-Pe, Theorem 3.1]. Then the proposition follows from Corollary 3.8 and Corollary 3.14

3.6. Remarks on higher dimensional case. Campana and Peternell [Ca-Pe] have made the following conjecture:

Conjecture 3.17. Suppose $X$ is a variety with a nef tangent bundle of dimension $d$. Then there is an étale cover $X^{\prime} \longrightarrow X$ such that $X^{\prime}$ admits a smooth fibration $X^{\prime} \longrightarrow A$ whose fibres are homogeneous spaces and $A$ is an abelian variety.

Furthermore, they also pose the following:

Question 3.18 ([Ca-Pe, p. 170]). Is every Fano manifold with a nef tangent bundle a homogeneous variety?

The above Conjecture 3.17 is proved in $\mathrm{Ca}-\mathrm{Pe}$ when $d \leq 3$. In higher dimensions, Demailly-Peternell-Schneider [DPS] prove that the fibres of $X^{\prime} \rightarrow A$ are Fano manifolds with a nef tangent bundle if $X$ is as above. An affirmative answer to the above question in all dimensions will prove Conjecture 3.17. If one can show the existence of a relative Chow-Künneth decomposition for the fibration $X^{\prime} \rightarrow A$ or show that it is a relative cellular variety, then the results of Proposition 3.16 will hold for all varieties which have a nef tangent bundle.

\section{ACKNOWLEDGEMENTS}

We are grateful to R. Joshua for asking the question to extend some results in Ak-Jo to other examples and for the hospitality during a visit in Oct. 2005. During the preparation of this article, he brought to our attention the motivic Hard Lefschetz theorem and issues on the existence of relative cellular decomposition and we are thankful to him. We also thank S-I. Kimura for explaining his work [Ki], S. Müller-Stach for his comments on the note and P. Deligne for pointing out some errors in the earlier version. The author is currently supported by the National Science Foundation under agreement No. DMS-0111298.

\section{REFERENCES}

[Ak-Jo] Akhtar, R., Joshua, R. Künneth decompositions for quotient varieties, Indagationes Math, 17, 3, 319-344, (2006) MR2321103

[Ak-Jo2] Akhtar, R., Joshua, R. Lefschetz decomposition for quotient varieties, to appear in $K$-theory.

[Be] Beauville, A. Sur l'anneau de Chow d'une variété abélienne, (French) [The Chow ring of an abelian variety] Math. Ann. 273 (1986), no. 4, 647-651. MR826463 (87g:14049) 
[Ca-Pe] Campana, F. and Peternell, T. Projective manifolds whose tangent bundles are numerically effective, Math. Ann. 289 (1991), 169-187. MR1087244 (91m:14061)

[Co-Ha] Corti, A., Hanamura, M. Motivic decomposition and intersection Chow groups. I, Duke Math. J. 103 (2000), no. 3, 459-522. MR.1763656 (2001f:14039)

[dA-Mü1] del Angel, P., Müller-Stach, S. Motives of uniruled 3-folds, Compositio Math. 112 (1998), no. 1, 1-16. MR.1622755 (99c:14004)

[dA-Mü2] del Angel, P., Müller-Stach, S. On Chow motives of 3-folds, Trans. Amer. Math. Soc. 352 (2000), no. 4, 1623-1633. MR1603890 (2000i:14005)

[DPS] Demailly, J.P, Peternell, T., Schneider, M. Compact complex manifolds with numerically effective tangent bundles, Journal of Algebraic Geometry 3 (1994), 295-345. MR.1257325 (95f:32037)

[De-Mu] Deninger, Ch., Murre, J. Motivic decomposition of abelian schemes and the Fourier transform, J. Reine Angew. Math. 422 (1991), 201-219. MR1133323 (92m:14055)

[Fu] Fulton, W. Intersection theory, Second edition. Ergebnisse der Mathematik und ihrer Grenzgebiete. 3. Folge., 2. Springer-Verlag, Berlin, 1998. xiv+470 pp. MR1644323 (99d:14003)

[La-Bi] Lange, H., Birkenhake, Ch. Complex abelian varieties, Grundlehren der Mathematischen Wissenschaften, 302, Springer-Verlag, Berlin, 1992. viii+435 pp. MR1217487 $(94 \mathrm{j}: 14001)$

[Go-Mu] Gordon, B., Murre, J. Chow motives of elliptic modular threefolds, J. Reine Angew. Math. 514 (1999), 145-164. MR1711275 (2001a:14005)

[GHM] Gordon, B. B., Hanamura, M., Murre, J.P. Relative Chow-Künneth projectors for modular varieties, J. Reine Angew. Math. 558 (2003), 1-14. MR1979179 (2004g:14008)

[GHM2] Gordon, B. B., Hanamura, M., Murre, J. P. Absolute Chow-Künneth projectors for modular varieties, J. Reine Angew. Math. 580 (2005), 139-155. MR2130589 (2006a:14006)

[Gu-Pe] Guletskiǔ, V., Pedrini, C. Finite-dimensional motives and the conjectures of Beilinson and Murre, Special issue in honor of Hyman Bass on his seventieth birthday. Part III. $K$-Theory 30 (2003), no. 3, 243-263. MR2064241 (2005f:14020)

[Ja] Jannsen, U. Motivic sheaves and filtrations on Chow groups, Motives (Seattle, WA, 1991), 245-302, Proc. Sympos. Pure Math., 55, Part 1, Amer. Math. Soc., Providence, RI, 1994. MR 1265533 (95c:14006)

[Ki] Kimura, S-I. Chow groups are finite dimensional, in some sense, Math. Ann. 331 (2005), no. 1, 173-201. MR2107443 (2005j:14007)

[Kl] Kleiman, S. L. Algebraic cycles and the Weil conjectures. Dix esposés sur la cohomologie des schémas, pp. 359-386. North-Holland, Amsterdam; Masson, Paris, 1968. MR0292838 (45:1920)

[Ko] Köck, B. Chow motif and higher Chow theory of $G / P$, Manuscripta Math. 70 (1991), 363-372. MR.1092142 (91m:14077)

[Kol] Kollár, J. Fundamental groups of rationally connected varieties, Mich. Math. Jour. 48 (2000) 359-368. MR1786496 (2001k:14045)

[KMM] Kollár, J., Miyaoka, Y., Mori, S. Rationally connected varieties, J. Algebraic Geometry 1 (1992) 429-448. MR:1158625 (93i:14014)

$[\mathrm{Ku}] \quad$ Künnemann, K. A. Lefschetz decomposition for Chow motives of abelian schemes, Invent. Math. 113 (1993), no. 1, 85-102. MR.1223225 (95d:14004)

[Ma] Macdonald, I.G. The Poincaré polynomial of a symmetric product, Proc. Cambridge Philos. Soc. 58, 1962, 563-568. MR0143204 (26:764)

[Man] Manin, Yu. Correspondences, motifs and monoidal transformations (in Russian), Mat. Sb. (N.S.) 77 (119) (1968), 475-507. MR0258836 (41:3482)

[MWYK] Miller, A., Müller-Stach, S., Wortmann, S., Yang, Y.H., Zuo, K. Chow-Künneth decomposition for universal families over Picard modular surfaces, Motives and Algebraic cycles I and II (eds. J. Nagel and Ch. Peters), London Math. Society Lecture Notes 343/344, Cambridge (2007).

[Mu1] Murre, J. P. On the motive of an algebraic surface, J. Reine Angew. Math. 409 (1990), 190-204. MR 1061525 (91g:14003)

[Mu2] Murre, J. P. On a conjectural filtration on the Chow groups of an algebraic variety. I. The general conjectures and some examples, Indag. Math. (N.S.) 4 (1993), no. 2, 177-188. MR 1225267 (94j:14006a) 
[Mu3] Murre, J. P. On a conjectural filtration on the Chow groups of an algebraic variety. II. Verification of the conjectures for threefolds which are the product on a surface and a curve, Indag. Math. (N.S.) 4 (1993), no. 2, 189-201. MR.1225268 (94j:14006b)

[Ne-Za] Nenashev, A., Zainoulline, K. Oriented cohomology and motivic decompositions of relative cellular spaces, J. Pure Appl. Algebra 205 (2006), no. 2, 323-340. MR 2203620 (2006i:14017)

[Sa] Saito, M. Chow-Künneth decomposition for varieties with low cohomological level, arXiv math.AG/0604254.

[Sc] Scholl, A. J. Classical motives, Motives (Seattle, WA, 1991), 163-187, Proc. Sympos. Pure Math., 55, Part 1, Amer. Math. Soc., Providence, RI, 1994. MR 1265529 (95b:11060)

[Sh] Shermenev, A.M. The motive of an abelian variety, Funct. Analysis, 8 (1974), 55-61. MR0335523 (49:304)

School of Mathematics, Institute for Advanced Study, 1 Einstein Drive, Princeton, NEW JERSEY 08540

E-mail address: jniyer@ias.edu

Current address: The Institute of Mathematical Sciences, CIT Campus, Taramani, Chennai 600113, India

E-mail address: jniyer@imsc.res.in 